\title{
Invasão biológica em sítios de Restinga no Nordeste brasileiro
}

\author{
Biological invasion in Restinga sites in northeastern Brazil \\ Invasión biológica en sitios de Restinga en el noreste de Brasil
}

Recebido: 10/05/2021 | Revisado: 17/05/2021 | Aceito: 21/05/2021 | Publicado: 07/06/2021

\author{
Juliano Ricardo Fabricante \\ ORCID: https://orcid.org/0000-0003-4767-7302 \\ Universidade Federal de Sergipe, Brasil \\ E-mail: julianofabricante@hotmail.com \\ Anny Bianca Santos Cruz \\ ORCID: https://orcid.org/0000-0002-1350-3702 \\ Universidade Federal de Sergipe, Brasil \\ E-mail: annybianca26@gmail.com \\ Francyelle Mathias dos Reis \\ ORCID: https://orcid.org/0000-0002-7025-8010 \\ Universidade Federal de Sergipe, Brasil \\ E-mail: francyellemathiasreis@gmail.com \\ Thieres Santos Almeida \\ ORCID: https://orcid.org/0000-0001-7371-8070 \\ Universidade Federal de Sergipe, Brasil \\ E-mail: thiieres@outlook.com
}

\begin{abstract}
Resumo
A Restinga é um importante ecossistema brasileiro que vem sendo acometido por problemas ambientais, a exemplo das invasões biológicas. Assim, objetivou-se com o presente trabalho realizar um levantamento das espécies naturalizadas e exóticas invasoras ocorrentes em sítios de Restinga no Nordeste brasileiro. Foram realizadas expedições em nove cidades de seis estados nordestinos. O levantamento ocorreu através de caminhadas (busca ativa) onde as espécies foram registradas por fotografias. Cada espécie foi classificada como naturalizada ou exótica invasora através de observações in situ e literatura específica. A composição de espécies das áreas foi comparada através da Similaridade de Jaccard $(S j)$ em três situações: lista completa, apenas as exóticas invasoras e apenas as naturalizadas. O teste de Mantel foi realizado para verificar se o tamanho e a distância entre áreas influenciaram nos resultados da similaridade. Foram amostradas 42 espécies, sendo 33 exóticas invasoras e nove naturalizadas. As espécies Leucena leucocephala (Lam.) de Wit, Terminalia catappa L. e Cenchrus echinatus L. foram as únicas com ocorrência em todas as áreas. A similaridade florística variou bastante entre as situações testadas. O teste de Mantel não apresentou valores significativos. Nossos achados são preocupantes, em razão do elevado número de espécies e da alta quantidade de exóticas invasoras.
\end{abstract}

Palavras-chave: Espécies alóctones; Exóticas invasoras; Litoral arenoso; Litoral nordestino.

\begin{abstract}
Restinga is an important Brazilian ecosystem that has been affected by environmental problems, such as biological invasions. Thus, we aim to carry out a survey of invasive or current alien species in Restinga in the Northeast of Brazil. The survey took place through walks where the species were recorded by photographs. Each species was classified as naturalized or invasive through in situ observations and specific literature. The species composition of the areas was compared using Jaccard Similarity $(S j)$ in three situations: complete list, only invasive and only naturalized. The Mantel test was performed to verify whether the size and distance between areas influence the similarity. 42 species were sampled, of which 33 were invasive and nine were naturalized. The Leucena leucocephala (Lam.) de Wit, Terminalia catappa L. and Cenchrus echinatus L. were the only ones occurring in all areas. The floristic similarity varied greatly between tested situations. Mantel test did not show significant values. Our findings are worrying, due to high number of species and high quantity of invasive species.
\end{abstract}

Keywords: Allochthonous species, Invasive species, Sandy coastline, Northeastern coast.

\section{Resumen}

Restinga es un importante ecosistema brasileño que se ha visto afectado por problemas ambientales, como invasiones biológicas. Por lo tanto, el objetivo de este estudio fue realizar un estudio de especies naturalizadas e exóticas invasoras presentes en los sitios de Restinga en el noreste de Brasil. Se realizaron expediciones a nueve ciudades del Noreste, en seis estados. La prospección se realizó a través de paseos donde se registraron las especies mediante fotografías. Cada especie fue clasificada como invasora naturalizada o exótica a través de observaciones in situ y literatura específica. La composición de especies de las áreas se comparó utilizando Jaccard Semejanza $(S j)$ en tres 
situaciones: lista completa, solo exóticas invasoras y solo naturalizadas. Se realizó la prueba de Mantel para verificar si el tamaño y la distancia entre áreas influyen en la similitud. Se muestrearon 42 especies, de las cuales 33 fueron invasoras y nueve fueron naturalizadas. Las especies Leucena leucocephala (Lam.) de Wit, Terminalia catappa L. y Cenchrus echinatus L. fueron las únicas que se presentaron en todas las áreas. La similitud florística varió mucho entre las situaciones probadas. La prueba de Mantel no mostró valores significativos. Nuestros hallazgos son preocupantes, debido a la gran cantidad de especies y la alta frecuencia de invasores exóticos.

Palabras clave: Especies foráneas; Exótico invasivo; Costa arenosa; Costa noreste.

\section{Introdução}

A Restinga é um ecossistema litorâneo sob influência marinha que apresenta grande biodiversidade (Assis, 2004). Esse ecossistema é muito importante na estabilização do substrato e redução dos efeitos de agentes erosivos (Assumpção \& Nascimento, 2000). As espécies que constituem a Restinga são adaptadas às condições adversas derivadas da proximidade com os mares e oceanos, a exemplo de ventos constantes, solos arenosos, baixos níveis de fertilidade do solo e elevado grau de salinidade (Barcelos et al., 2012).

A colonização do território brasileiro iniciou-se pela região litorânea (Azevedo, 1994), resultando no adensamento populacional observado atualmente. De acordo com IBGE (2010), vivem nas cidades litorâneas a maior parte da população brasileira. Em razão disso, a Restinga encontra-se intensamente modificada e sob forte pressão antrópica (Thomazi et al., 2013). Essas condições, por sua vez, favorecem as invasões biológicas, uma vez que ambientes alterados são mais susceptíveis a ocorrência desses processos (Zenni \& Ziller, 2011).

As invasões biológicas (IB) ocorrem quando espécies exóticas são introduzidas em um novo ambiente, se adaptam e passam a se reproduzir e a se dispersar para novas áreas modificando os ecossistemas naturais (Parker et al. 1999). As IB geram efeitos negativos sobre a biota nativa (Wolfe \& Klironomos, 2005; Reinhart \& Callaway, 2006), o ambiente físico (D’antonio \& Vitousek, 1992; Wolfe \& Klironomos, 2005; Ziller \& Zalba, 2007; Fabricante et al., 2012), além de causar prejuízos econômicos (Pacheco \& Marinis, 1984; Taylor \& Irwin, 2004).

Devido a importância da Restinga e aos impactos gerados pelas invasões biológicas, objetivou-se com o presente trabalho realizar um levantamento das espécies naturalizadas e exóticas invasoras ocorrentes em sítios de Restinga no Nordeste brasileiro.

\section{Metodologia}

\subsection{Locais de estudo}

O presente estudo foi realizado em nove cidades de seis estados do Nordeste brasileiro (Figura 1). Informações detalhadas sobre os locais podem ser obtidas na Tabela 1. 
Figura 1: Locais estudados. Sendo: I = Salvador, BA; II = Mata de São João, BA; III = Entre Rios, BA; IV = Esplanada, BA; V = Aracaju, SE; VI = Maceió, AL; VII = Tamandaré, PE; VIII = João Pessoa, PB; IX = Natal, RN.

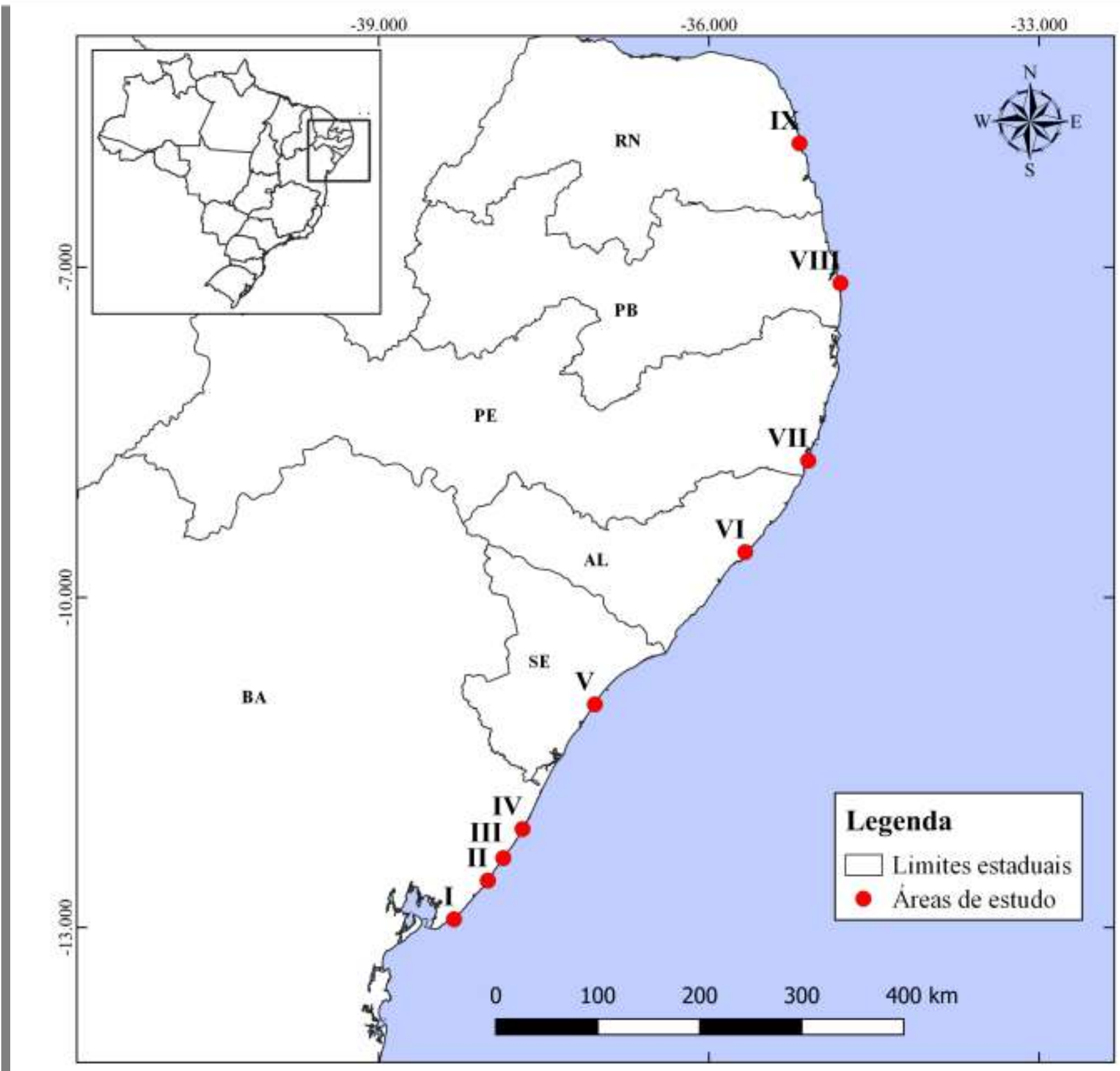

Fonte: Autores (2021). 
Tabela 1: Informações sobre as localidades estudadas. Sendo: Af = quente e úmido; Am = tropical de monção; As = tropical com estação seca.

\begin{tabular}{lcccc}
\hline Municípios/UF & Localidades & Coordenadas & $\begin{array}{c}\text { Distância } \\
\text { percorrida }\end{array}$ & Clima \\
\hline $\begin{array}{l}\text { Salvador/BA } \\
\text { Mata de São }\end{array}$ & Praia do Flamengo e Aleluia & $12^{\circ} 55^{\prime} 36.72^{\prime \prime} \mathrm{S} ; 38^{\circ} 18^{\prime} 59.91^{\prime \prime} \mathrm{W}$ & $3 \mathrm{~km}$ & Af \\
João/BA & Praia do Forte & $12^{\circ} 34^{\prime} 29,89^{\prime \prime} \mathrm{S} ; 38^{\circ} 00^{\prime} 33,60^{\prime \prime} \mathrm{W}$ & $2 \mathrm{~km}$ & Af \\
Entre Rios/BA & Porto do Sauipe & $12^{\circ} 22^{\prime} 14.42^{\prime \prime} \mathrm{S} ; 37^{\circ} 52^{\prime} 8.87^{\prime \prime} \mathrm{W}$ & $1,5 \mathrm{~km}$ & Af \\
Esplanada/ & Praia do Baixio & $12^{\circ} 6^{\prime} 23.11^{\prime \prime} \mathrm{S} ; 37^{\circ} 41^{\prime} 37.51^{\prime \prime} \mathrm{W}$ & $0,06 \mathrm{~km}$ & Am \\
BA & Praia da Aruana e Atalaia & $10^{\circ} 58^{\prime} 25,52^{\prime \prime} \mathrm{S} ; 37^{\circ} 2^{\prime} 11,29^{\prime \prime} \mathrm{W}$ & $3,8 \mathrm{~km}$ & Am \\
Aracaju/SE & Praia Cruz das Almas, & $9^{\circ} 35^{\prime} 21,63 " \mathrm{~S} ; 35^{\circ} 40^{\prime} 12,70^{\prime \prime} \mathrm{W}$ & $3,8 \mathrm{~km}$ & Am \\
Maceió/AL & Jacarecica do Sul e Guaxuma & $8^{\circ} 45^{\prime} 30.1^{\prime \prime} \mathrm{S} ; 35^{\circ} 05^{\prime} 48.9^{\prime \prime} \mathrm{W}$ & $0,9 \mathrm{~km}$ & Am \\
Tamandaré/PE & Praia de Tamandaré & $7^{\circ} 8^{\prime} 44,20^{\prime \prime} \mathrm{S} ; 34^{\circ} 48^{\prime} 15,69^{\prime \prime} \mathrm{W}$ & $12 \mathrm{~km}$ & As \\
João Pessoa/PB & Praia de Cabo Branco e Manaíra & & \\
Natal/RN & Praia de Mãe Luiza e Ponta & $5^{\circ} 52^{\prime} 27,78^{\prime \prime} \mathrm{S} ; 35^{\circ} 10^{\prime} 35,17^{\prime \prime} \mathrm{W}$ & $8,4 \mathrm{~km}$ & As \\
\hline
\end{tabular}

Fonte: Autores (2021); Clima (Alvares et al., 2013).

\subsection{Coleta e análise de dados}

O levantamento foi realizado por meio de caminhadas (busca ativa) pelas áreas estudadas, onde todas as espécies encontradas foram registradas por meio de fotografias. Foram realizadas ao menos duas expedições para cada município entre os anos de 2016 e 2020. A determinação das espécies ocorreu por meio de consultas a especialistas e a literatura especializada.

As espécies não nativas foram classificadas em naturalizadas ou exóticas invasoras por meio de observação in situ e por consulta às bases de dados online (CABI, 2021; Base de Dados Nacional de Espécies Exóticas Invasoras, Instituto Hórus de Desenvolvimento e Conservação Ambiental; 2021, ISSG, 2021) e artigos científicos sobre o tema invasão biológica. Foram consideradas naturalizadas as espécies exóticas que se reproduzem e formam populações viáveis apenas próximo a área na qual foram introduzidas (Richardson et al., 2000). Já as exóticas invasoras, as espécies exóticas com capacidade de se dispersar e invadir locais distantes da sua área de introdução inicial (Moro et al., 2012).

Para avaliar a semelhança florística entre as áreas estudadas foram realizadas análises de similaridade de Jaccard ( $\mathrm{Sj}$ ). Essas análises foram feitas em três diferentes condições: i) utilizando a lista geral de espécies; ii) utilizando apenas as exóticas invasoras e; iii) utilizando apenas as naturalizadas. Para verificar se o tamanho das áreas amostrais e ou a distância espacial entre elas influenciaram no resultado das análises de similaridade, foram realizadas análises de Mantel com 9.999 randomizações. A significância considerada no teste foi de $p<0,05$. As avaliações foram efetuadas utilizando o pacote “vegan” (Oksanen et al., 2020) no software R (R Core Team, 2021).

\section{Resultados e Discussão}

Foram inventariadas 42 espécies não nativas, das quais 33 exóticas invasoras e nove naturalizadas (Tabela 2; Figura 2; Figura 3; Figura 4). A família que apresentou o maior número de espécies foi Poaceae (30,95\%), seguida por Fabaceae $(9,52 \%)$ e Asparagaceae, Apocynaceae, Cucurbitaceae e Euphorbiaceae (7,14\%, cada uma). As famílias restantes apresentaram apenas uma espécie cada. 
Tabela 2: Espécies naturalizadas $(\mathrm{N})$ e exóticas invasoras (EI) inventariadas nas áreas estudadas. Sendo: 1 = presença da espécie; 0 = ausência da espécie; I = Salvador, BA; II = Mata de São João, BA; III = Entre Rios, BA; IV = Esplanada, BA; V = Aracaju, SE; VI = Maceió, AL; VII = Tamandaré, PE; VIII = João Pessoa, PB; IX = Natal, RN.

\begin{tabular}{lllllllllll}
\hline Família/Espécie & I & II & III & IV & V & VI & VII & VIII & IX & Status \\
\hline ACANTHACEAE & & & & & & & & & &
\end{tabular}

Asystasia gangetica (L.) T.Anderson

$\begin{array}{llllllllll}1 & 0 & 0 & 0 & 0 & 0 & 0 & 0 & 0 & \text { EI }\end{array}$

AMARANTHACEAE

Amaranthus viridis L.

$\begin{array}{llllllllll}0 & 0 & 0 & 1 & 1 & 1 & 0 & 1 & 1 & \text { EI }\end{array}$

ANACARDIACEAE

Mangifera indica $\mathrm{L}$.

APOCYNACEAE

Calotropis procera (Aiton) W.T.Aiton

Catharanthus roseus (L.) Don

Cryptostegia madagascariensis Bojer

ASPARAGACEAE

Agave angustifolia Haw.

Agave sisalana Perrine ex Engelm

Sansevieria trifasciata Prain

ASTERACEAE

Cosmos sulphureus Cav.

CACTACEAE

Opuntia dillenii (Ker Gawl.) Haw

CASUARINACEAE

Casuarina equisetifolia L.

\section{COMBRETACEAE}

Terminalia catappa $\mathrm{L}$.

\section{CUCURBITACEAE}

Citrullus lanatus (Thunb.) Matsum. \& Nakai

Luffa cylindrica (L.) M.Roem.

Momordica charantia L.

CYPERACEAE

Cyperus rotundus $\mathrm{L}$.

EUPHORBIACEAE

Euphorbia cyathophora Murray

Jatropha gossypiifolia L.

Ricinus communis L.

FABACEAE

Crotalaria pallida Aiton

Crotalaria retusa $\mathrm{L}$.

Leucaena leucocephala (Lam.) de Wit

Pithecellobium dulce (Roxb.) Benth.

$\begin{array}{llllllllll}0 & 0 & 1 & 0 & 0 & 0 & 0 & 0 & 0 & \text { EI } \\ 0 & 0 & 0 & 0 & 1 & 1 & 0 & 0 & 0 & \text { EI } \\ 0 & 0 & 0 & 1 & 1 & 1 & 0 & 1 & 0 & \text { EI }\end{array}$

$\begin{array}{llllllllll}0 & 1 & 1 & 1 & 0 & 1 & 0 & 1 & 1 & \text { EI } \\ 0 & 1 & 0 & 1 & 0 & 1 & 0 & 0 & 0 & \mathrm{~N} \\ 0 & 0 & 0 & 0 & 0 & 1 & 0 & 0 & 1 & \mathrm{~N}\end{array}$

$\begin{array}{llllllllll}1 & 0 & 0 & 1 & 0 & 1 & 0 & 1 & 1 & \mathrm{~N} \\ 1 & 1 & 0 & 0 & 0 & 0 & 1 & 0 & 0 & \mathrm{~N} \\ 0 & 0 & 0 & 0 & 0 & 0 & 0 & 0 & 1 & \mathrm{~N}\end{array}$

$\begin{array}{llllllllll}0 & 0 & 0 & 0 & 0 & 0 & 0 & 1 & 0 & \text { EI }\end{array}$

$\begin{array}{llllllllll}1 & 1 & 1 & 0 & 1 & 1 & 0 & 1 & 1 & \mathrm{~N}\end{array}$

$\begin{array}{llllllllll}1 & 1 & 1 & 1 & 1 & 1 & 1 & 1 & 1 & \text { EI }\end{array}$




\begin{tabular}{|c|c|c|c|c|c|c|c|c|c|c|}
\hline Família/Espécie & $\mathbf{I}$ & II & III & IV & $\mathbf{V}$ & VI & VII & VIII & $\mathbf{I X}$ & Status \\
\hline \multicolumn{11}{|l|}{ MALVACEAE } \\
\hline Thespesia populnea (L.) Sol. Ex Corrêa & 1 & 0 & 0 & 0 & 0 & 1 & 0 & 0 & 0 & EI \\
\hline \multicolumn{11}{|l|}{ MYRTACEAE } \\
\hline Syzygium cumini (L.) Skeels & 1 & 0 & 0 & 1 & 0 & 1 & 1 & 1 & 0 & EI \\
\hline \multicolumn{11}{|l|}{ NYCTAGINACEAE } \\
\hline Boerhavia diffusa $\mathrm{L}$. & 1 & 0 & 0 & 0 & 1 & 1 & 0 & 1 & 1 & EI \\
\hline \multicolumn{11}{|l|}{ POACEAE } \\
\hline Arundo donax $\mathrm{L}$. & 0 & 0 & 0 & 0 & 0 & 1 & 0 & 0 & 0 & $\mathrm{~N}$ \\
\hline Cenchrus echinatus $\mathrm{L}$. & 1 & 1 & 1 & 1 & 1 & 1 & 1 & 1 & 1 & EI \\
\hline Cenchrus purpureus (Schumach.) Morrone & 0 & 0 & 0 & 0 & 0 & 1 & 0 & 0 & 0 & EI \\
\hline Cynodon dactylon (L.) Pers. & 1 & 1 & 1 & 0 & 1 & 1 & 1 & 1 & 1 & EI \\
\hline Dactyloctenium aegyptium (L.) Willd. & 1 & 1 & 0 & 1 & 1 & 1 & 0 & 1 & 1 & EI \\
\hline Echinochloa colona (L.) Link & 0 & 0 & 0 & 0 & 1 & 1 & 0 & 0 & 0 & EI \\
\hline Eleusine indica (L.) Gaertn. & 0 & 0 & 0 & 0 & 1 & 1 & 0 & 0 & 0 & EI \\
\hline Eragrostis ciliaris (L.) R.Br. & 0 & 1 & 0 & 0 & 1 & 0 & 0 & 0 & 0 & EI \\
\hline $\begin{array}{l}\text { Eragrostis tenella (L.) P.Beauv. ex Roem. \& } \\
\text { Schult. }\end{array}$ & 0 & 1 & 0 & 1 & 0 & 1 & 0 & 0 & 0 & EI \\
\hline $\begin{array}{l}\text { Megathyrsus maximus (Jacq.) B.K.Simon \& } \\
\text { S.W.L.Jacobs }\end{array}$ & 1 & 1 & 0 & 0 & 1 & 1 & 0 & 1 & 1 & EI \\
\hline Melinis repens (Willd.) Zizka & 0 & 1 & 0 & 0 & 0 & 1 & 0 & 1 & 0 & EI \\
\hline $\begin{array}{l}\text { Sorghum bicolor subsp. arundinaceum (Desv.) de } \\
\text { Wet \& J.R.Harlan }\end{array}$ & 0 & 0 & 0 & 0 & 1 & 1 & 1 & 0 & 0 & EI \\
\hline $\begin{array}{l}\text { Urochloa brizantha (Hochst. ex A. Rich.) } \\
\text { R.D.Webster }\end{array}$ & 1 & 0 & 0 & 0 & 0 & 1 & 0 & 0 & 0 & EI \\
\hline \multicolumn{11}{|l|}{ POLYGONACEAE } \\
\hline Antigonon leptopus Hook. \& Arn. & 1 & 0 & 0 & 0 & 0 & 1 & 0 & 0 & 0 & EI \\
\hline \multicolumn{11}{|l|}{ RUBIACEAE } \\
\hline Morinda citrifolia $\mathrm{L}$. & 0 & 0 & 0 & 0 & 1 & 0 & 0 & 0 & 0 & EI \\
\hline
\end{tabular}


Research, Society and Development, v. 10, n. 6, e48410615942, 2021

(CC BY 4.0) | ISSN 2525-3409 | DOI: http://dx.doi.org/10.33448/rsd-v10i6.15942

Figura 2: Espécies exóticas invasoras e naturalizadas inventariadas nas Restingas do Nordeste brasileiro.

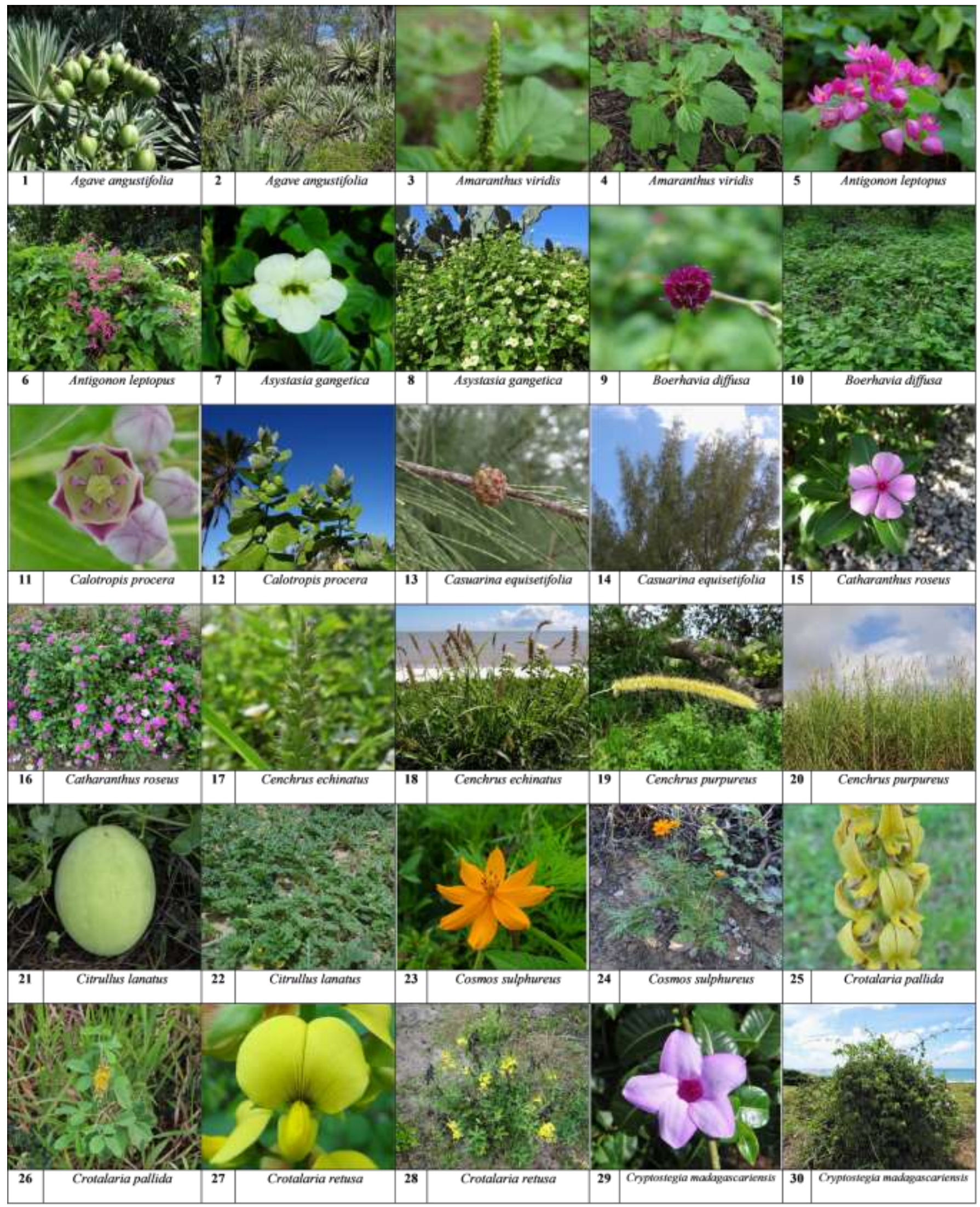

Fonte: Autores (2021). 
Research, Society and Development, v. 10, n. 6, e48410615942, 2021

(CC BY 4.0) | ISSN 2525-3409 | DOI: http://dx.doi.org/10.33448/rsd-v10i6.15942

Figura 3: Espécies exóticas invasoras e naturalizadas inventariadas nas Restingas do Nordeste brasileiro.

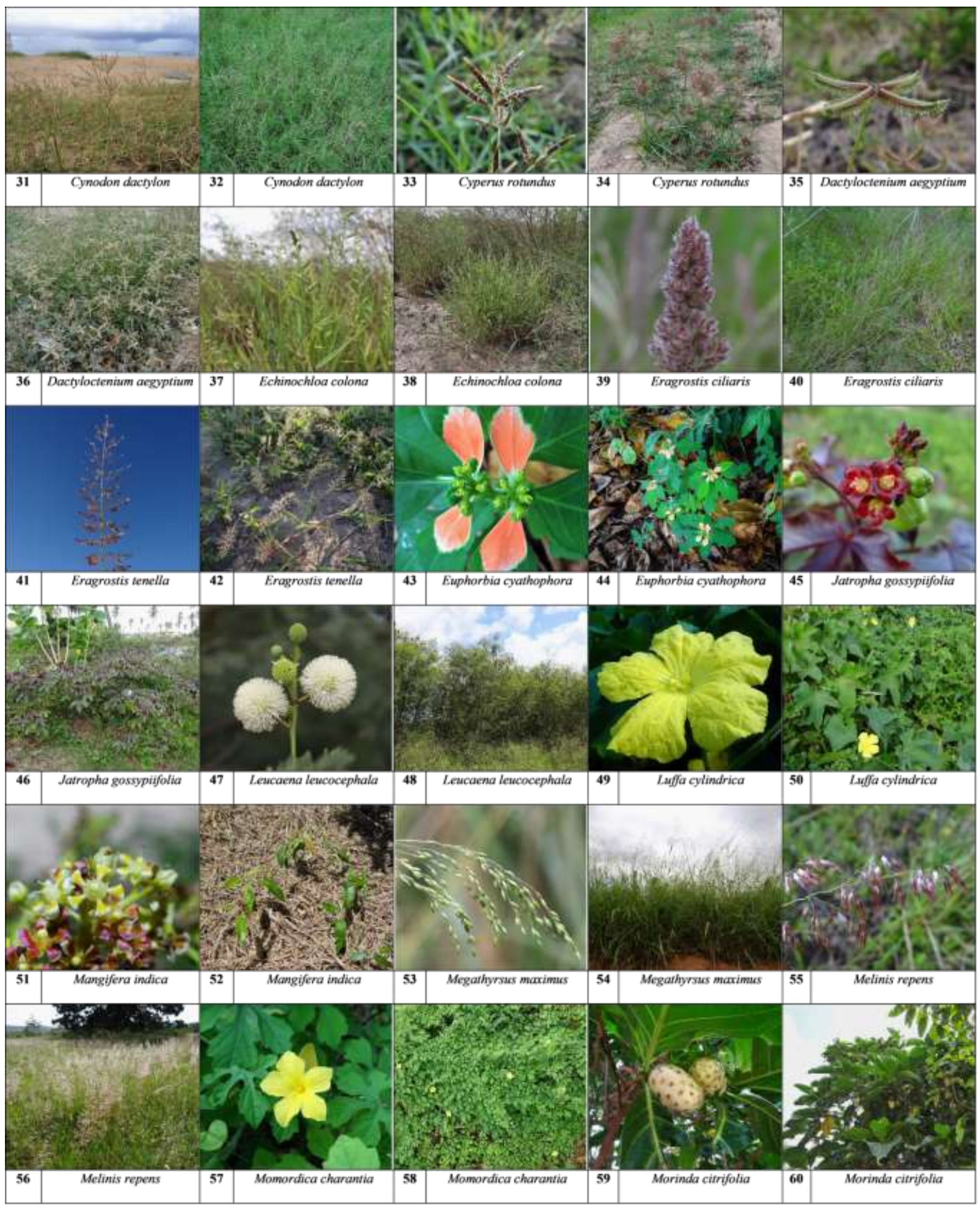

Fonte: Autores (2021). 
Figura 4: Espécies exóticas invasoras e naturalizadas inventariadas nas Restingas do Nordeste brasileiro.

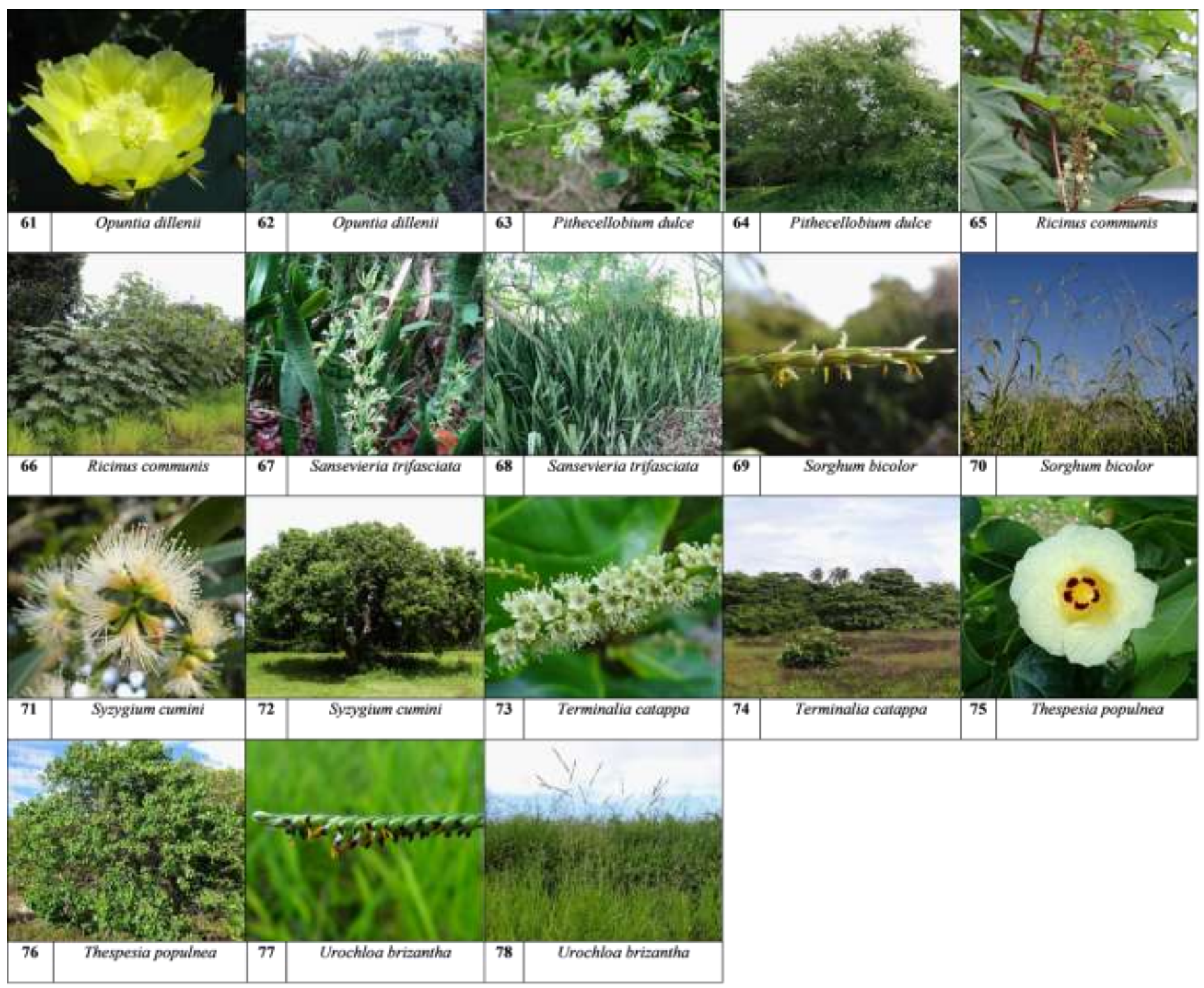

Fonte: Autores (2021).

A riqueza de plantas naturalizadas e exóticas invasoras encontrada nas áreas estudadas é preocupante, uma vez que é superior a obtida em estudos desenvolvidos em outros biomas/ecossistemas brasileiros (Quartarola et al., 2015; Fabricante et al., 2015; Silva \& Fabricante, 2019; Alves \& Fabricante, 2019; Araújo et al., 2021). Essa alta riqueza pode ser justificada pelas condições de conservação desse ecossistema. É notório que a pressão antrópica sobre a Restinga é intensa e ocorre desde a chegada dos Europeus ao Brasil (Azevedo, 1994; Thomazi, et al., 2013), gerando ambientes favoráveis ao estabelecimento de processos de invasão biológica. Muitos estudos (Pegado et al., 2006; Hulme, 2009; Guerra et al., 2018) já estabeleceram essa relação.

A proporção entre o número de espécies exóticas invasoras e naturalizadas encontrada é semelhante a alguns outros trabalhos (Quartarola et al., 2015; Silva \& Fabricante, 2019; Araújo et al., 2021). Em todos há a predominância de espécies exóticas invasoras quando comparada as naturalizadas. A capacidade dessas espécies (exóticas invasoras) de se dispersar para novas áreas sem ajuda humana devem explicar esses resultados (Richardson et al., 2000; Moro et al., 2012).

É importante ressaltar que espécies naturalizadas podem progredir, no futuro, para a condição de exóticas invasoras (Richardson et al., 2000). Essa progressão pode ocorrer graças a introdução em múltiplos ambientes e ao aumento da 
variabilidade genética (Perez, 2006). Além disso, as mudanças climáticas também podem atuar nesse processo (Hellman et al., 2008).

As espécies mais frequentes foram Leucena leucocephala (Lam.) de Wit, Terminalia catappa L. e Cenchrus echinatus L., registradas em todas as áreas (100\%). Cynodon dactylon (L.) Pers. e Jatropha gossypiifolia L. foram amostradas em oito áreas (88,9\%). Opuntia dillenii (Ker Gawl.) Haw, Ricinus communis L. e Dactyloctenium aegyptium (L.) Willd. em sete áreas (77,8\%). Catharanthus roseus (L.) Don, Agave sisalana Perrine ex Engelm, Melinis repens (Willd.) Zizka e Sorghum bicolor subsp. arundinaceum (Desv.) de Wet \& J.R.Harlan em três áreas (33,3\%). Mangifera indica L., Cryptostegia madagascariensis Bojer, Luffa cylindrica (L.) M.Roem., Crotalaria pallida Aiton, Thespesia populnea (L.) Sol. Ex Corrêa, Urochloa brizantha (Hochst. ex A. Rich.) R.D.Webster e Antigonon leptopus Hook. \& Arn. foram registras em duas áreas (22,2\%). Por fim, as espécies Cosmos sulphureus Cav, Euphorbia cyathophora Murray, Arundo donax L., Cenchrus purpureus (Schumach.) Morrone e Morinda citrifolia L. foram observadas em apenas uma área (11,1\%).

As espécies mais frequentes possuem características intrínsecas que justificam suas amplitudes de ocorrência. A espécie L. leucocephala é uma das 100 exóticas invasoras mais agressivas do mundo (Lowe, Browne, Boudjelas \& Poorter, 2000). Ela é extremamente resistente, resiliente e produz altas quantidades de sementes durante o ano todo (Base de Dados Nacional de Espécies Exóticas Invasoras, Instituto Hórus de Desenvolvimento e Conservação Ambiental, 2021). A espécie $T$. catappa, por sua vez, forma maciços populacionais capazes de excluir as espécies nativas por meio de competição por recursos e por interferência (Plucênio et al., 2013; Santos \& Fabricante, 2018; Base de Dados Nacional de Espécies Exóticas Invasoras, Instituto Hórus de Desenvolvimento e Conservação Ambiental, 2021). Já C. echinatus, é uma espécie que consegue se dispersar através de diversos vetores e se estabelecer em diferentes ambientes (Base de Dados Nacional de Espécies Exóticas Invasoras, Instituto Hórus de Desenvolvimento e Conservação Ambiental, 2021). Além disso, é considerada uma das seis espécies mais agressivas da agricultura moderna (Pacheco \& Martins, 1984).

A similaridade, considerando todas as espécies, variou de 0,22 a 0,64 , sendo a mais baixa entre as áreas VI e VII e a mais alta entre as áreas VIII e IX (Tabela 3; Figura 5.A). Considerando apenas as exóticas invasoras, a similaridade variou de 0,24 a 0,67, sendo a mais baixa entre as áreas III e VI e a mais alta entre as áreas VIII e IX (Tabela 3; Figura 5.B). Já considerando somente as naturalizadas, a similaridade variou de 0 a 0,67 , sendo nula entre várias áreas e a mais alta entre as áreas I e VIII (Tabela 3; Figura 5.C).

Os resultados obtidos nas análises de similaridade demonstram que a composição florística pouco varia em todo o litoral estudado, especialmente quando considerado somente as espécies exóticas invasoras. Especula-se que esses resultados se devem ao elevado potencial de dispersão das exóticas invasoras que as possibilita colonizar novos ambientes além da área de introdução (ver Richardson et al., 2000).

O teste de Mantel indicou não haver dependência espacial nos resultados das análises de similaridade (total - $r=-0,24$, $\mathrm{p}=0,92$; exóticas invasoras $-\mathrm{r}=-0,26, \mathrm{p}=0,95$; naturalizadas $-\mathrm{r}=-0,16, \mathrm{p}=0,80$ ). Segundo o mesmo teste, o tamanho das áreas também não influenciou nesses resultados (total $-r=-0,19, p=0,79$; exóticas invasoras $-r=-0,24, p=0,87$; naturalizadas $-r=-0,05, p=0,53$ ). 
Tabela 3: Similaridade de Jaccard (matrizes). A similaridade foi realizada em três situações: utilizando a lista completa das espécies, apenas as espécies exóticas invasoras e apenas as naturalizadas. Sendo: I = Salvador, BA; II = Mata de São João, BA; III= Entre Rios, BA; IV = Esplanada, BA; V = Aracaju, SE; VI = Maceió, AL; VII = Tamandaré, PE; VIII = João Pessoa, PB; IX = Natal, RN.

\begin{tabular}{|c|c|c|c|c|c|c|c|c|}
\hline \multicolumn{9}{|c|}{ Lista completa } \\
\hline & $\bar{I}$ & II & III & IV & $\overline{\mathbf{V}}$ & VI & VII & VIII \\
\hline II & 0,42 & & & & & & & \\
\hline III & 0,26 & 0,44 & & & & & & \\
\hline IV & 0,37 & 0,42 & 0,30 & & & & & \\
\hline $\mathrm{V}$ & 0,37 & 0,41 & 0,30 & 0,31 & & & & \\
\hline VI & 0,50 & 0,42 & 0,23 & 0,50 & 0,53 & & & \\
\hline VII & 0,36 & 0,35 & 0,27 & 0,23 & 0,24 & 0,22 & & \\
\hline VIII & 0,60 & 0,48 & 0,32 & 0,54 & 0,46 & 0,54 & 0,30 & \\
\hline IX & 0,50 & 0,43 & 0,39 & 0,38 & 0,42 & 0,43 & 0,24 & 0,64 \\
\hline \multicolumn{9}{|c|}{ Exóticas invasoras } \\
\hline II & 0,41 & & & & & & & \\
\hline III & 0,25 & 0,47 & & & & & & \\
\hline IV & 0,39 & 0,45 & 0,35 & & & & & \\
\hline V & 0,38 & 0,43 & 0,29 & 0,36 & & & & \\
\hline VI & 0,55 & 0,45 & 0,24 & 0,54 & 0,57 & & & \\
\hline VII & 0,39 & 0,38 & 0,33 & 0,28 & 0,29 & 0,29 & & \\
\hline VIII & 0,59 & 0,52 & 0,30 & 0,57 & 0,48 & 0,59 & 0,37 & \\
\hline IX & 0,53 & 0,53 & 0,43 & 0,42 & 0,48 & 0,43 & 0,33 & 0,67 \\
\hline \multicolumn{9}{|c|}{ Naturalizadas } \\
\hline II & 0,50 & & & & & & & \\
\hline III & 0,33 & 0,33 & & & & & & \\
\hline IV & 0,25 & 0,25 & 0,00 & & & & & \\
\hline V & 0,25 & 0,25 & 0,50 & 0,00 & & & & \\
\hline VI & 0,29 & 0,29 & 0,17 & 0,33 & 0,33 & & & \\
\hline VII & 0,25 & 0,25 & 0,00 & 0,00 & 0,00 & 0,00 & & \\
\hline VIII & 0,67 & 0,25 & 0,50 & 0,33 & 0,33 & 0,33 & 0,00 & \\
\hline IX & 0,40 & 0,17 & 0,25 & 0,20 & 0,20 & 0,43 & 0,00 & 0,50 \\
\hline
\end{tabular}


Figura 5: Similaridade de Jaccard (clusters). A similaridade foi realizada em três situações: utilizando a lista completa das espécies (A), apenas as espécies exóticas invasoras (B) e apenas as naturalizadas (C). Sendo: I = Salvador, BA; II = Mata de São João, BA; III= Entre Rios, BA; IV = Esplanada, BA; V = Aracaju, SE; VI = Maceió, AL; VII = Tamandaré, PE; VIII = João Pessoa, PB; IX = Natal, RN.

A B

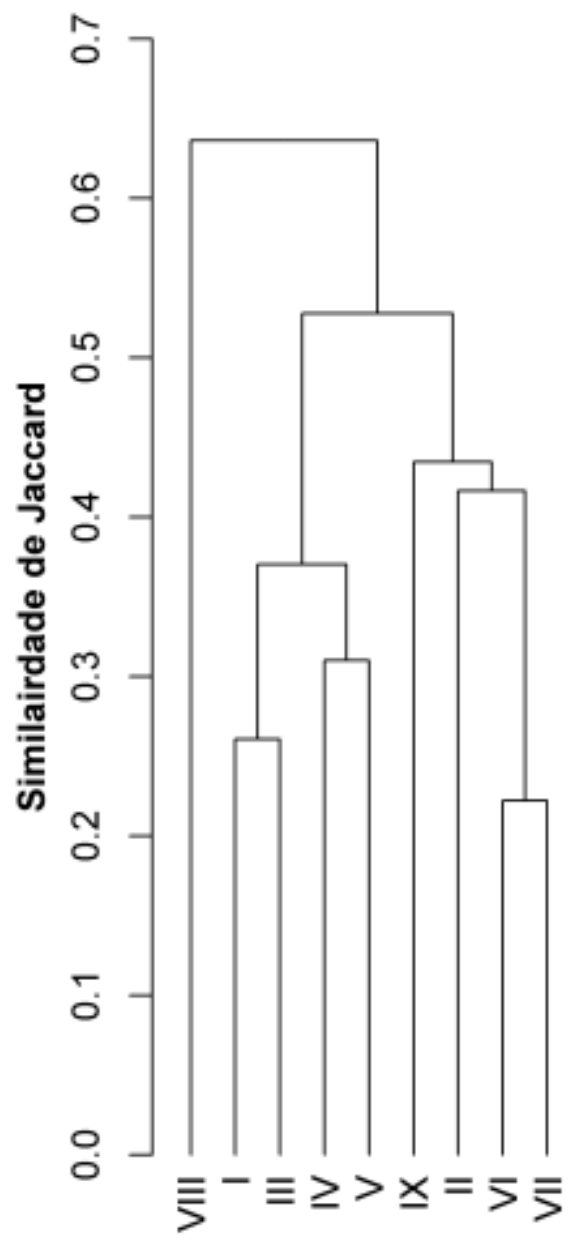

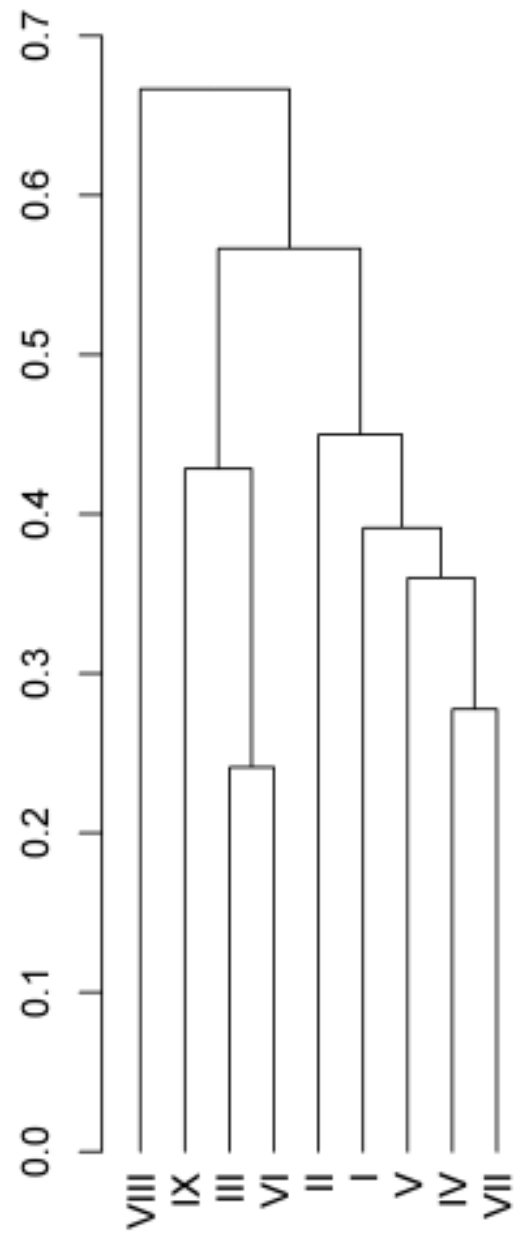

C

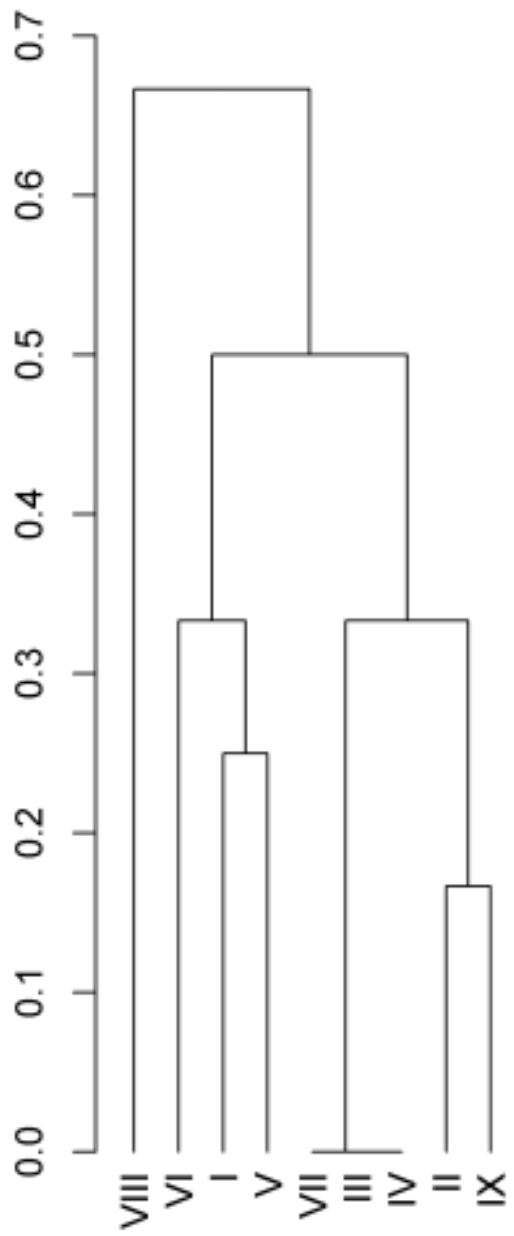

Fonte: Autores (2021).

\section{Conclusão}

A riqueza de exóticas invasoras e naturalizadas encontrada nos sítios de Restinga estudados foi elevada. Dentre as espécies amostradas, várias são consideradas extremamente agressivas, capazes de causar impactos ambientais e econômicos. Desta forma, nossos resultados sugerem que é necessário e urgente o controle das espécies não nativas inventariadas e a recuperação dos sítios estudados.

\section{Referências}

Alvares, C. A., Stape, J. L., Sentelhas, P. C., Gonçalvez, J. D., \& Sparovek, G. (2013). Köppen's climate classification map for Brazil. Meteorologische Zeitschrift, 22(6), 711-728.

Alves, J. S. \& Fabricante, J. R. (2019). Exotic Invasive Flora Evaluation on Different Environments and Preservation Conditions from an Area Belonging to Caatinga, Petrolina, PE. Gaia Scientia, 13(1), 140-152.

Araújo, K. C. T., Cruz, A. B. S. \& Fabricante, J. R. (2021). Invasão Biológica na Área de Proteção Ambiental Morro do Urubu, Aracaju, Sergipe, Brasil. Revista de Biologia e Ciências da Terra, 21, 72-82, 2021. 
Assis A. M, Thomaz, L. D. \& Pereira, O. J. (2004). Florística de um trecho de restinga no município de Guarapari, Espírito Santo, Brasil. Acta Botanica Brasilica, 18(1), 191-201.

Assumpção, J. \& Nascimento, M. T. (2000). Estrutura e composição florística de quatro formações vegetais de restinga no complexo lagunar Grussaí/Iquipari, São João da Barra, RJ, Brasil. Acta Botanica Brasilica, 14(3), 158-164.

Azevedo, A. (1994). Vilas e cidades do Brasil Colonial. Ensaio de Geografia Urbana Retrospectiva. Terra Livre. São Paulo, Associação dos Geógrafos Brasileiros, (10), 23-78.

Barcelos, M. E., Riguete, J. R., Silva, L. T. \& Ferreira Jr, P. F. (2012). Uma visão panorâmica sobre os solos das restingas e seu papel na definição de comunidades vegetais nas planícies costeiras do sudeste do Brasil. Natureza online, 10(2), 71-76.

Base de Dados Nacional de Espécies Exóticas Invasoras, Instituto Hórus de Desenvolvimento e Conservação Ambiental. http://bd.institutohorus.org.br.

CABI. (2021). https://www.cabi.org/.

CENSO, IBGE. (2021). http://www.censo2010.ibge.gov.br/.

D'antonio, C. \& Vitousek, P. M. (1992). Biological invasions by exotic grasses, the grass fire cycle, and global change. Annual Review of Ecology and Systematics, 23(1), $63-87$.

Fabricante, J. R., Araújo, K. C. T., Andrade, L. A. \& Ferreira, J. V. A. (2012). Invasão Biológica de Artocarpus heterophyllus Lam. (Moraceae) em um Fragmento de Mata Atlântica no Nordeste do Brasil: Impactos sobre a Fitodiversidade e os Solos dos Sítios Invadidos. Acta Botanica Brasílica, 26(2), 399407.

Fabricante, J. R., Ziller, S. R., Araújo, K. C. T., Furtado, M. D. D. G. \& Arantes, B. F. (2015). Non-native and invasive alien plants on fluvial islands in the São Francisco River, northeastern Brazil. Check List, 11(1), 1535.

Guerra, C., Baquero, R. A., Guttiérrez-Arellano, D. \& Nicola, G. G. (2018). Is the Natura 2000 network effective to prevent the biological invasions?. Global Ecology and Conservation, 18(1), 2351-9894.

Hellmann, J. J, Byers, J., Britta G. Bierwagen, B. G. \& Dukes, J. S. (2008). Five Potential Consequences of Climate Change for Invasive Species. Conservation Biology. 22(3), 534-543.

Hulme, P. E. (2009). Trade, transport and trouble: managing invasive species pathways in an era of globalization. Journal of applied ecology, 46(1), 10-18.

ISSG. (2021). Invasive Species Specialist Group. http://www.issg.org/.

Lowe, S., Browne, M., Boudjelas, S. \& Poorter, M. (2000). 100 of the world's worst invasive alien species: a selection from the global invasive species database. The Invasive Species Specialist Group/Species Survival Commission/ World Conservation Union IUCN, 1(1), 11.

Moro, M. F., Souza, V. C., Oliveira-filho, A. T. D., Queiroz, L. P. D., Fraga, C. N. D., Rodal, M. J. N., Araujo, F. S. \& Martins, F. R. (2012). Alienígenas na sala: o que fazer com espécies exóticas em trabalhos de taxonomia, florística e fitossociologia? Acta Botanica Brasilica, 26(4), 991-999.

Oksanem, F. J., Blanchet, G., Friendly, M., Kindt, R., Legendre, P., MC Glinn, D., Minchin, P. R., O'hara, R. B., Simpson, G. L., Solymos, P., Stevens, M. H. H., Szoecs, E. \& Wagner, H. (2020). Community Ecology Package. R package version, 2(1), 5-7.

Pacheco, R. P. B. \& Marntis, G. (1984). Ciclo de vida, estruturas reprodutivas e dispersão de populações experimentais de capim-carrapicho (Cenchrus echinatus L.). Planta Daninha,7(1),13-21.

Parker, I. M., Simberloff, D., Lonsdale, W. M., Goodell, K., Wonham, M., Kareiva, P. M., Williamson, M. H., Von Holle, B., Moyle, P. B., Byers, J. E. \& Goldwasser, L. (1999). Impact: toward a framework for understanding the ecological effects of invaders. Biological invasions, 1(1), 3-19.

Pegado, C. M. A., Andrade, L. A. D., Félix, L. P. \& Pereira, I. M. (2006). Efeitos da invasão biológica de algaroba: Prosopis juliflora (Sw.) DC. sobre a composição e a estrutura do estrato arbustivo-arbóreo da caatinga no Município de Monteiro, PB, Brasil. Acta Botanica Brasilica, 20 (4), 887-898.

Perez, J. E. (2006). The biology of invasions: the genetic adaptation paradox. Biological Invasions, 8(5), 1115-1121.

Plucênio, R. M., Dechoum, M. S. \& Castellani, T. T. (2013). Invasão Biológica em Restinga: O Estudo de caso de Terminalia catappa L. (Combretaceae). Biodiversidade Brasileira, 3(2), 118-136.

Quartarola, H. L., Defensor, B. R., Barbosa, B. B. \& Morais, T. P. S. (2015). Levantamento fitossociológico de plantas invasoras na recuperação de áreas degradadas no Parque Estadual do Pau Furado, no Triângulo Mineiro. Semana de Estudos de Engenharia Ambiental, 3(1), 44-52.

R Core Team (2020) R: A language and environment for statistical computing. R Foundation for Statistical Computing, Vienna. Version 4.0.0. https://www.R-project.org/.

Reinhart, K. \& Callaway, R. (2006). Soil biota and invasive plants. The New phytologist, 170(3), 445-457.

Richardson, D. M., Pysek, P., Rejmánek, M., Barbour, M. G., Panetta, F. D. \& West, C. J. (2000). Naturalization and invasion of alien plants: concepts and definitions. Diversity and distributions, 6(2), 93-107.

Santos, J. P. B. \& Fabricante, J. R. (2018). Population structure and effects by the invasive exotic indian-almond over autochthonous vegetation from a sandbank. Neotropical Biology and Conservation, 13(4), 295-302.

Silva, F. O. \& Fabricante, J. R. (2019). Invasão Biológica no Parque Nacional do Catimbau, Pernambuco, Brasil. Revista de Ciências Ambientais, 13(2), 1726. 
Research, Society and Development, v. 10, n. 6, e48410615942, 2021

(CC BY 4.0) | ISSN 2525-3409 | DOI: http://dx.doi.org/10.33448/rsd-v10i6.15942

Taylor, B. W. \& Irwvin, R. E. (2004). Linking economic activities to the distribution of exotic plants. Proceedings of the National Academy of Sciences, 101(51), 17725-17730.

Thomazi, R. D., Rocha, R. T., Oliveira, M. V., Bruno, A. S. \& Silva, A. G. (2013). Um panorama da vegetação das restingas do Espírito Santo no contexto do litoral brasileiro, Natureza on line, 11(1), 1-6.

Wolfe, B. E. \& Klironomos, J. N. (2005) Breaking new ground: soil communities and exotic plant invasion. Bioscience, 55(6), 477-487.

Zenni, R. D. \& Ziller, S. R. (2011). An overview of invasive plants in Brazil. Revista Brasileira de Botânica, 34(3), 431-446.

Ziller, S. R. \& Zalba, S. (2007). Propostas de ação para prevenção e controle de espécies exóticas invasoras. Natureza \& Conservação, 5(2), 8-15. 